Such decisions are frequently challenging to make, and may be contested within an organization. They also have enormous ramifications for the communities who are receiving assistance. Methods: Drawing on field cases of project closure, and an examination of the literature around this topic, we conducted a normative and conceptual analysis of humanitarian organizations' obligations.

Results: Humanitarian projects have intrinsic, as well as instrumental value, and thus create moral entanglements between humanitarian actors and local communities that require careful consideration due to the responsibilities that ensue. Basing our proposal on an analysis of the nature of relationships between providers and recipients of humanitarian aid, we argue that ethical exit strategies should reflect five commitments. In closing projects, humanitarian organizations should demonstrate respect for recipients of care, and seek to minimize harm and disruption by acting in ways that are characterized by: (1) transparency, (2) predictability, (3) adaptability, (4) participation, and (5) evaluation. In addition, humanitarian organizations have responsibilities toward their staff who will implement the closure of a project. These responsibilities include ensuring that relevant policies and resources are in place, and that training and support are provided to those who require it.

Conclusion: Closing projects is an inescapable aspect of humanitarian action - indeed, almost all humanitarian projects will come to an end. Making and enacting such decisions is ethically fraught, and may be a source of distress for humanitarian workers and local communities. Careful attention to ethical exit strategies that follow through on obligations toward local communities is therefore a vital component of ethical humanitarian action.

Prehosp Disaster Med 2017;32(Suppl. 1):s47-s48

doi:10.1017/S1049023X17001376

\section{Ethical Challenges of Providing End of Life Care in an Ebola Treatment Unit (2014-2015) \\ Heather Draper}

Division Of Health Sciences, University of Warwick, West Midlands/ United Kingdom

Study/Objective: To identify and explore the ethical challenges faced by UK Defence Medical Services personnel working in an Ebola Treatment Unit (ETU) established in Sierra Leone.

Background: British military were deployed as part of the UK Government's response to the Ebola outbreak in West Africa (Op GRITROCK). This included establishing a small, wellresourced ETU for Ebola-infected international responders and local healthcare workers. The End of Life Care (EoLC) provision in humanitarian disaster response is discussed very little in bioethics.

Methods: Twenty personnel who deployed between October 2014 and April 2015 were interviewed about the ethical challenges they faced. Participants included doctors (7), nurses (6) and other healthcare related (7). A largely conventional approach to content analysis was taken using the data to draw conclusions about themes in the participants' thinking. Data was organized using NVivo. Only the EoLC theme is reported here.
Results: EoLC caused particular ethical difficulties, even though most participants were accustomed to dealing with the dying and dead. Specific issues included: uncertainty about the course of the disease in individuals, which resulted in, amongst other things, a 'hybrid' approach to palliation; the trade-off between infection control and providing 'normal' end of life care and comfort; moving dying patients long distances to receive palliative care; preparation of the dead for burial. The duty to care for patients ('normal' professional obligations) was constrained by public health measures (containment) and the need to protect staff from infection (obligations to employees/self/ colleagues). End of life care, where human contact seems especially important, was particularly impacted.

Conclusion: Meaningful end of life care is difficult to provide during a mass outbreak of a highly infectious and serious disease. The difficulties of providing meaningful care need to be taken into account when deciding how to prioritize and deliver EoLC in a disaster response.

Prehosp Disaster Med 2017;32(Suppl. 1):s48

doi:10.1017/S1049023X17001388

Ethical Challenges at the Intersection of Policy and Practice in Humanitarian Contexts

Leigh-Anne Gillespie ${ }^{1}$, Lisa Schwartz ${ }^{1}$, Julia Abelson ${ }^{1}$, Lydia Kapiriri ${ }^{2}$, Matthew Hunt ${ }^{3}$

1. Clinical Epidemiology And Biostatistics, McMaster University, Hamilton/ON/Canada

2. Health, Aging \& Society, McMaster University, Hamilton/ON/ Canada

3. School Of Physical And Occupational Therapy, McGill University, Montreal/QC/Canada

Study/Objective: To explore how policies and ethics inform each other, in order to better understand where problems arise in humanitarian healthcare organizations, and how policy can be improved in this regard.

Background: Researchers have just begun to understand the range of ways in which humanitarian healthcare organizations' policies can shape ethical dilemmas in the field. This study lays out some of the ethical tensions that affect the profession by identifying ethical challenges that arise from aid agency policies and practices, or that trigger the need for improved policy development.

Methods: We interviewed 14 Organizational Members (OMs) from Canadian and international offices of humanitarian healthcare organizations to explore the questions and objectives guiding our study. In order to understand how problems and ethical issues are identified in humanitarian aid organizations, and their relationship to policies and practices in this regard, we used interpretive description (ID) methodology. We prioritize understanding to go beyond description and more deeply into the interpretive aspect of ID, in order to unpack problems and ethical issues in humanitarian aid.

Results: Three key themes were identified: participant perceptions of tensions related to 1) institutional memory loss; 2) priorities within different departments of humanitarian healthcare organizations; and, 3) social norms and expectations of humanitarian healthcare organizations and local communities. 
Conclusion: It is important for humanitarians to identify and discuss ethical challenges and problems, to ensure responses to emergencies are not disconnected or lead to negative impacts. While this paper cannot show saturation of the types of ethical challenges facing humanitarian healthcare organizations, it is a move to bring stories forward and formalize and capture histories so we can learn from them.

Prehosp Disaster Med 2017;32(Suppl. 1):s48-s49

doi:10.1017/S1049023X1700139X

Aid - When There is "Nothing Left to Offer": A Survey and Qualitative Study of Ethics and Palliative Care During

International Humanitarian Emergencies

Lisa Schwartz ${ }^{1}$, Matthew Hunt ${ }^{2}$, Elysee Nouvet ${ }^{3}$,

Humanitarian Health Ethics Research Group Hhe ${ }^{1}$

1. Clinical Epidemiology And Biostatistics, McMaster University, Hamilton/ON/Canada

2. School Of Physical And Occupational Therapy, McGill University, Montreal/QC/Canada

3. Clinical Epidemiology And Biostatistics, McMaster University, Hamilton/Canada

Study/Objective: How can humanitarian organizations support ethically and contextually appropriate palliative care in humanitarian crises? This ELRHA (R2HC) funded study explores the ethical complexities of doing so, asking how existing standards of palliative care may be adapted to support delivery of ethically and contextually appropriate palliative care in humanitarian action.

Background: There is a lack of evidence clarifying ethical and practical possibilities and consequences of humanitarian organizations, addressing or failing to address patients' palliative needs. This study seeks to inform realistic, context-sensitive guidance, education, and practice for the provision of palliative care during humanitarian emergencies. Beginning with a survey of international aid organizations, we aim to identify a baseline of current palliative care provisions for clinical and psychosocial care in humanitarian action.

Methods: Concurrent exploratory mixed-methods involving 1) survey to investigate to what extent humanitarian organizations enable staff to provide palliative care, 2) interviews with stakeholders (local/expatriate humanitarian staff, local care providers, community members) to better understand lived experiences of palliative care needs in humanitarian emergencies.

Results: Survey results and preliminary analysis of interviews will be shared. Responses cover: preparedness to deliver palliative care in humanitarian emergencies (disasters, conflict areas, epidemics); resources currently available to support the delivery of palliative care in humanitarian contexts, and ideas/concerns related to integration of palliative care into humanitarian healthcare.

Conclusion: Palliative care is an area of growing global concern. It is increasingly recognized as necessary, yet simultaneously seen as outside the realm of possibility, particularly in humanitarian settings, where care in life threatening conditions may be logistically and ethically challenging. The 67th World Health Assembly resolved on "strengthening palliative care as a component of comprehensive care throughout the life course."
Our study helps illuminate ethical and practical concerns for applying palliative care in humanitarian crisis.

Prehosp Disaster Med 2017;32(Suppl. 1):s49

doi:10.1017/S1049023X17001406

Developing the Disaster Medical Assistant Team Education and Training Program (DMAT)

Soon-Joo Wang

Hallym University, Hwaseong/Republic of Korea

Study/Objective: The study objective is to create the contents and program in disaster medical training system.

Background: Disaster medical education and training were not mandatory in Korea, but several kinds of programs existed. The disaster medical education and training has been mandatory in Korea since 2014. The newly developed official disaster medical education and training system, which should be applied from 2015, was necessary.

Methods: We reviewed the disaster medical education and training program in advanced countries including the US, EU and Japan, and compared them with the existing programs in Korea. After analysis and comparison, a new contents and program for Korean situations was developed, and they were applied to the pilot program repeatedly for confirmation of adequate educational effect.

Results: First, the official national disaster medical manual was developed, and the contents and programs were developed after that. The program includes the following;

- disaster medical system

- Disaster Medical Assistance Team (DMAT) operation

- logistics

- sample activities of DMAT

- radiologic disaster

- chemical disaster

- disaster mental health support

- communication system in disaster

- in hospital disaster

- equipment preparedness for EMAT

- table top simulation

- comprehensive disaster drill The pilot program operated appropriately.

Conclusion: For medical response in a disaster, the standard minimal requirement for disaster medical education and training should be developed, and disaster medical personnel should participate in this program.

Prehosp Disaster Med 2017;32(Suppl. 1):s49

doi:10.1017/S1049023X17001418

Vulnerable Populations: Investigating Ethical Implications for Policies and Practices of International Humanitarian Organizations

Lisa Eckenwiler ${ }^{1}$,Ayesha Abmad ${ }^{2}$, Ryoa Chung ${ }^{3}$, Matthew Hunt ${ }^{4}$, Jackie Leach Scully ${ }^{5}$, Lisa Schwarz ${ }^{6}$, Nina Wild

1. Philosophy, George Mason University, Fairfax/VA/United States of America

2. University College London, London/United Kingdom 